\title{
Regularity of Linear Systems of Differential Equations on the Axes and Pencils of Quadratic Forms
}

\author{
Viktor Kulyk ${ }^{1}$, Ganna Kulyk², Nataliia Stepanenko ${ }^{3 *}$
}

\begin{abstract}
It is considered linear systems of differential equations and investigated questions of regularity of these systems. To explore the regularity it is comfortable to use quadratic form whose derivative with respect to the adjoint system is positive definite. Sometimes it is possible to find such a quadratic form, the derivative of which with respect to the system is non-negative. There are examples showing that in this case we can't say anything about the exponential dichotomy of this system (that is, its regularity). The question arises whether it is possible to combine a certain set of quadratic forms to get such a form, the derivative of which with respect to the system is positive definite. This question is similar to the question that arises in the theory of control: having a set of certain data about an object, can one say something about this object as a whole. It turns out that this is possible, only a set of these quadratic forms should be special, in some sense complete. In the presented article the authors propose to write it with the help of some combination of specific symmetric matrices $S_{1}, S_{2}, \ldots$. So we have a quadratic form
\end{abstract}

$$
V_{p}=p_{1}\left\langle S_{1}(t) x, x\right\rangle+p_{2}\left\langle S_{2}(t) x, x\right\rangle+\cdots+p_{k-1}\left\langle S_{k-1}(t) x, x\right\rangle+\left\langle S_{k}(t) x, x\right\rangle
$$

It is proved that the derivative of this quadratic form is positive definite for sufficiently large parameters $p_{1}, \ldots, p_{k-1}$. The results are illustrated by examples.

Keywords: Adjoint system, Quadratic form, Regular, Symmetric matrices, Weakly regular 2010 AMS: Primary 34A30

1 Silesian University of Technology, Kaszubska 23, 44-102 Gliwice, Poland, email: viktor.kulyk@polsl.pl

${ }^{2}$ National Technical University of Ukraine "Igor Sikorsky Kyiv Polytechnic Institute", Peremogy avenue 37, 03056 Kyiv, Ukraine, email: ganna_1953@ukr.net

${ }^{3}$ National Technical University of Ukraine "Igor Sikorsky Kyiv Polytechnic Institute", Peremogy avenue 37, 03056 Kyiv, Ukraine, email: nataliya.stepanenko@III.kpi.ua, ORCID:0000-0002-9690-4797

*Corresponding author: nataliya.stepanenko@III.kpi.ua

Received: 7 April 2019, Accepted: 3 July 2019, Available online: 30 September 2019

\section{Introduction}

In many interesting investigations [1]-[4] it is arisen linear systems of differential equations in which we have to find the strong properties, i.e. such properties which arent changed under small perturbations. Such properties often are exponential dichotomy and trichotomy of the solutions of linear systems of differential equations. As for non-stationary systems this question is opened it is interesting to find something new in investigation of dichotomy of linear systems of differential equations. The investigation 
of sets of quadratic forms is a promising and relevant topic, since they provide an opportunity to answer the question of the magnitude of perturbation, which does not disturb the property of the regularity of linear systems. Consequently, we consider certain classes of systems of linear equations and try to find quadratic forms that will enable us to investigate these systems.

\section{Main results}

Let's consider the homogeneous system of differential equations

$$
\frac{d x}{d t}=P(t) x,
$$

where $x \in R^{n}$ and $P(t)$ is $n \times n$-dimensional matrix with scalar functions whose elements are real continuous and bounded on $R=(-\infty,+\infty)$. We will denote by $C^{0}(R)$ the space of functions which are continuous and bounded on $R$ and by $C^{1}(R)-$ the subspace of space $C^{0}(R)$ of continuously differentiable functions with bounded derivative on $R$.

We will consider a norm of a vector $x \in R^{n}$ as $\|x\|=\sqrt{\langle x, x\rangle}$, where $\langle x, y\rangle=\sum_{j=1}^{n} x_{j} y_{j}$-scalar product in $R^{n}$. And we will denote a norm of a matrix $A$ as $\|A\|=\max \|A x\|,\|x\|=1,\|A\|_{0}=\sup \|A(t)\|, t \in R$.

The important question about system (2.1) is its regularity on entire axis $R$. It is known the following definition of regularity [1]:

Definition 2.1. The system (2.1) is called regular on $R$ if corresponding non-homogeneous system $\frac{d x}{d t}=P(t) x+f(t)$ has unique bounded solution on $R$ with any fixed vector function $f(t) \in C^{0}(R)$. If it is only known that such system has at least one solution bounded on $R$ with any $f(t) \in C^{0}(R)$ then the system (2.1) is called weakly regular on $R$.

It is known that the system (2.1) is regular on $R$ if and only if there exists a quadratic form $V=\langle S(t) x, x\rangle$ where $S(t) \in C^{1}(R)$ - symmetric matrix whose derivative with respect to the system (2.1) is positive definite, i.e.

$$
\dot{V}=\left\langle\left[\frac{d S(t)}{d t}+S(t) P(t)+P^{T}(t) S(t)\right] x, x\right\rangle \geq\|x\|^{2}
$$

and wherein the matrix $S(t)$ is non-degenerated for any $t \in R$

$$
\operatorname{det} S(t) \neq 0 \quad \forall t \in R .
$$

In case the matrix $P(t)$ from the system (2.1) is a constant, from a weak regularity always follows the regularity. It can be if and only if real parts of all eigenvalues of matrix $P$ are non-zeroes. Therefore, if $\operatorname{det} P=0$ then the system (2.1) with constant matrix $P$ is not regular. It turns out that there exists variable matrix $P(t)$ such that $\operatorname{det} P(t) \equiv 0 \forall t \in R$ but the system (2.1) is regular on $R$. The examples of such systems are:

$$
\left\{\begin{array}{l}
\frac{d x_{1}}{d t}=x_{1}\left(p_{1} \cos 2 \omega t+p_{2} \sin 2 \omega t\right)+x_{2}\left(-p_{2} \cos 2 \omega t+p_{1} \sin 2 \omega t-\omega\right), \\
\frac{d x_{2}}{d t}=x_{1}\left(-p_{2} \cos 2 \omega t+p_{1} \sin 2 \omega t+\omega\right)-x_{2}\left(p_{1} \cos 2 \omega t+p_{2} \sin 2 \omega t\right)
\end{array}\right.
$$

where parameters $p_{1}, p_{2}, \omega \in R$ are non-zero, real and $p_{1}^{2}+p_{2}^{2}=\omega^{2}$.

In this case the derivative of non-degenerated quadratic form

$$
V=x_{1}^{2} \cos 2 \omega t+2 x_{1} x_{2} \sin 2 \omega t-x_{2}^{2} \cos 2 \omega t
$$

with respect to this system equals $\dot{V}=2 p_{1}\left(x_{1}^{2}+x_{2}^{2}\right)$.

Remark 2.2. For some systems (2.1) there exists symmetric matrices $S(t) \in C^{1}(R)$ which satisfy inequality (2.2) but the condition (2.3) is not satisfied. Then the system (2.1) is not regular but adjoint system $\frac{d x}{d t}=-P^{T}(t) x$ is weakly regular.

Linear operator $S(t) \in C^{1}(R)$ which affects on symmetric matrices we will denote $L[S]$ :

$$
L[S]=\frac{d S(t)}{d t}+S(t) P(t)+P^{T}(t) S(t)
$$

Remark 2.3. If instead of the inequality (2.2) we write $\langle L[S] x, x\rangle \geq\|N x\|^{2}$, where $N$ - some constant non-generated matrix, then we can't say anything about regularity of the system (2.1). We can see this from the example:

$$
\frac{d x_{1}}{d t}=x_{2}, \frac{d x_{2}}{d t}=0
$$

The derivative of quadratic form $V=x_{1} x_{2}$ with respect to this system is $\dot{V}=x_{2}^{2}$, but this system is not regular. 
The question arises: If we have not a single matrix $S$, but some set of matrices $S_{1}, S_{2}, \ldots$, then is it possible to combine these matrices to construct the matrix $S$ that satisfy the inequality $\langle L[S] x, x\rangle \geq\|x\|^{2}$ ? This article is devoted to investigating this question.

Theorem 2.4. Let there exists two matrices $S_{1}(t), S_{2}(t) \in C^{1}(R)$ which satisfy the following inequalities

$$
\left\{\begin{array}{c}
\left\langle L\left[S_{1}\right] M x, M x\right\rangle \geq \varepsilon_{1}\|(M-N) x\|^{2} \\
\left\langle L\left[S_{2}\right] N x, N x\right\rangle \geq \varepsilon_{2}\|N x\|^{2}, \quad \varepsilon_{1}, \varepsilon_{2}=\text { const }>0
\end{array}\right.
$$

for some constant matrices $M, N$. Then the sum of these matrices $\bar{S}=p S_{1}(t)+S_{2}(t)$ satisfies an inequality

$$
\langle L[S] M x, M x\rangle \geq \sigma(p)\|M x\|^{2},
$$

where $\sigma(p)=\frac{\left(p-\alpha \varepsilon_{1}\right) \varepsilon_{2}-\alpha^{2}}{2\left(p-\alpha \varepsilon_{1}+\varepsilon_{2}\right)},\left(p-\alpha \varepsilon_{1}\right) \varepsilon_{2}-\alpha^{2}>0$, constant $\alpha$ is chosen from inequality $\left\|L\left[S_{2}\right]\right\| \leq \alpha$.

Proof. Taking into account the linearity of the operator (2.4), we can write the left side of the inequality (2.6) in the following form:

$$
\langle L[\bar{S}] M x, M x\rangle=p\left\langle L\left[S_{1}\right] M x, M x\right\rangle+\left\langle L\left[S_{2}\right] M x, M x\right\rangle=p\left\langle L\left[S_{1}\right] M x, M x\right\rangle+\left\langle L\left[S_{2}\right] N x, N x\right\rangle+Q,
$$

where

$$
\begin{aligned}
Q & =\left\langle L\left[S_{2}\right] M x, M x\right\rangle-\left\langle L\left[S_{2}\right] N x, N x\right\rangle \\
& =\left\langle L\left[S_{2}\right] M x, M x\right\rangle-\left\langle L\left[S_{2}\right] M x, N x\right\rangle+\left\langle L\left[S_{2}\right] M x, N x\right\rangle-\left\langle L\left[S_{2}\right] N x, N x\right\rangle \\
& =\left\langle L\left[S_{2}\right] M x,(M-N) x\right\rangle+\left\langle L\left[S_{2}\right] N x,(M-N) x\right\rangle \\
& =\left\langle L\left[S_{2}\right](M-N+N) x,(M-N) x\right\rangle+\left\langle L\left[S_{2}\right] N x,(M-N) x\right\rangle \\
& =\left\langle L\left[S_{2}\right](M-N) x,(M-N) x\right\rangle+2\left\langle L\left[S_{2}\right] N x,(M-N) x\right\rangle .
\end{aligned}
$$

From this we obtain an estimation from below

$$
Q \geq-\alpha\|(M-N) x\|^{2}-2 \alpha\|N x\|\|(M-N) x\|^{2} .
$$

Therefore using inequalities (2.5) and (2.8) from inequality (2.7), we get

$$
\langle L[\bar{S}] M x, M x\rangle \geq\left(p-\alpha \varepsilon_{1}\right)\|(M-N) x\|^{2}-2 \alpha\|N x\|\|(M-N) x\|+\varepsilon_{2}\|N x\|^{2} .
$$

Let us write the quadratic form corresponding to the right side of the inequality (2.9)

$$
V\left(x_{1}, x_{2}\right)=\left(p-\alpha \varepsilon_{1}\right) x_{1}^{2}-2 \alpha x_{1} x_{2}+\varepsilon_{2} x_{2}^{2} .
$$

We should find its lowest value on a single circle $x_{1}=\cos y, x_{2}=\sin y$. We obtain

$V(\cos y, \sin y)=\left(p-\alpha \varepsilon_{1}\right) \frac{1+\cos 2 y}{2}-\alpha \sin 2 y+\varepsilon_{2} \frac{1-\cos 2 y}{2} \geq \frac{p-\alpha \varepsilon_{1}+\varepsilon_{2}}{2}-\sqrt{\left(\frac{p-\alpha \varepsilon_{1}-\varepsilon_{2}}{2}\right)^{2}+\alpha^{2}}=\frac{\left(p-\alpha \varepsilon_{1}\right) \varepsilon_{2}-\alpha^{2}}{\frac{p-\alpha \varepsilon_{1}+\varepsilon_{2}}{2}+\sqrt{\left(\frac{p-\alpha \varepsilon_{1}-\varepsilon_{2}}{2}\right)^{2}+\alpha^{2}}}$.

Choosing sufficiently large the value of parameter $p>0$, exactly $p>\alpha \varepsilon_{1}+\frac{\alpha^{2}}{\varepsilon_{2}}$, we get

$$
V\left(x_{1}, x_{2}\right) \geq \frac{\left(p-\alpha \varepsilon_{1}\right) \varepsilon_{2}-\alpha^{2}}{p-\alpha \varepsilon_{1}+\varepsilon_{2}}\left(x_{1}^{2}+x_{2}^{2}\right) \text {. }
$$

Therefore, from (2.9) we obtain

$$
\langle L[\bar{S}] M x, M x\rangle \geq \frac{\left(p-\alpha \varepsilon_{1}\right) \varepsilon_{2}-\alpha^{2}}{p-\alpha \varepsilon_{1}+\varepsilon_{2}}\left(\|(M-N) x\|^{2}+\|N x\|^{2}\right) .
$$

As for any matrices $M$ and $N$ of equal dimension the inequality

$$
\|(M-N) x\|^{2}+\|N x\|^{2} \geq \frac{1}{2}\|M x\|^{2}
$$


is fulfilled then from (2.10) we get

$$
\langle L[\bar{S}] M x, M x\rangle \geq \frac{\left(p-\alpha \varepsilon_{1}\right) \varepsilon_{2}-\alpha^{2}}{2\left(p-\alpha \varepsilon_{1}+\varepsilon_{2}\right)}\|M x\|^{2} .
$$

Theorem 2.5. Let there exists symmetric matrices $S_{j}(t) \in C^{1}(R), j=\overline{1, k}, n \times n$-dimensional and they satisfy the following inequalities:

$$
\begin{aligned}
& \left\langle L\left[S_{j}\right] M_{j}(t) x, M_{j}(t) x\right\rangle \geq\left\|\left[M_{j}(t)-M_{j+1}(t)\right] x\right\|^{2}, \quad j=\overline{1,(k-1)} \\
& \left\langle L\left[S_{k}\right] M_{k}(t) x, M_{k}(t) x\right\rangle \geq\left\|M_{k}(t) x\right\|^{2},
\end{aligned}
$$

with some $n \times n$-dimensional continuous non-degenerated matrices $M_{j}(t)$. Then the derivative of a quadratic form

$$
V_{p}=p_{1}\left\langle S_{1}(t) x, x\right\rangle+p_{2}\left\langle S_{2}(t) x, x\right\rangle+\cdots+p_{k-1}\left\langle S_{k-1}(t) x, x\right\rangle+\left\langle S_{k}(t) x, x\right\rangle
$$

with respect to the system (2.1) will be positive definite for sufficiently large fixed values of parameters $p_{1}, p_{2}, \ldots, p_{k-1}$.

Proof. Let us choose and fix the constant $\alpha$, that satisfy an inequalities $\left\|L\left[S_{j}\right]\right\| \leq \alpha, j=\overline{1, k}$. From the last of the inequalities (2.11)

$$
\left\langle L\left[S_{k-1}\right] M_{k-1}(t) x, M_{k-1}(t) x\right\rangle \geq\left\|\left[M_{k-1}(t)-M_{k}(t)\right] x\right\|^{2}
$$

and (2.12) using Theorem 2.4 with $\varepsilon_{1}=1, \varepsilon_{2}=1$ we get an inequality

$$
\left\langle L[\tilde{S}] M_{k-1}(t) x, M_{k-1}(t) x\right\rangle \geq \sigma\left(p_{k-1}\right)\left\|M_{k-1}(t) x\right\|^{2},
$$

where

$$
\tilde{S}=p_{k-1} S_{k-1}(t)+S_{k}(t), \sigma\left(p_{k-1}\right)=\frac{p_{k-1}-\alpha-\alpha^{2}}{2\left(p_{k-1}-\alpha+1\right)} .
$$

Then let us consider the penultimate of the inequalities (2.11)

$$
\left\langle L\left[S_{k-2}\right] M_{k-2}(t) x, M_{k-2}(t) x\right\rangle \geq\left\|\left[M_{k-2}(t)-M_{k-1}(t)\right] x\right\|^{2} .
$$

Together with the inequality (2.13), based on the Theorem $2.4\left(\varepsilon_{1}=1, \varepsilon_{2}=\sigma\left(p_{k-1}\right)\right)$ for the sum of the matrices

$$
\bar{S}=p_{k-2} S_{k-2}(t)+\tilde{S}=p_{k-2} S_{k-2}(t)+p_{k-1} S_{k-1}(t)+S_{k}(t),
$$

we get an inequality

$$
\left\langle L[\bar{S}] M_{k-2}(t) x, M_{k-2}(t) x\right\rangle \geq \sigma\left(p_{k-2}, p_{k-1}\right) \cdot\left\|M_{k-2}(t) x\right\|^{2},
$$

where

$$
\sigma\left(p_{k-2}, p_{k-1}\right)=\frac{\left(p_{k-2}-\alpha\right) \sigma\left(p_{k-1}\right)-\alpha^{2}}{2\left(p_{k-2}-\alpha+\sigma\left(p_{k-1}\right)\right)} .
$$

So we have the following estimation

$$
\begin{aligned}
& \left\langle L[\widehat{S}] M_{k-3}(t) x, M_{k-3}(t) x\right\rangle \geq \sigma\left(p_{k-3}, p_{k-2}, p_{k-1}\right)\left\|M_{k-3}(t) x\right\|^{2}, \\
& \sigma\left(p_{k-3}, p_{k-2}, p_{k-1}\right)=\frac{\left(p_{k-3}-\alpha\right) \sigma\left(p_{k-2}, p_{k-1}\right)-\alpha^{2}}{2\left(p_{k-2}-\alpha+\sigma\left(p_{k-2}, p_{k-1}\right)\right)} .
\end{aligned}
$$


Continuing to receive similar estimates, in the end for the sum of the matrices

$$
S_{p}=p_{1} S_{1}(t)+p_{2} S_{2}(t)+\cdots+p_{k-1} S_{k-1}(t)+S_{k}(t),
$$

we will get

$$
\left\langle L\left[S_{p}\right] M_{1}(t) x, M_{1}(t) x\right\rangle \geq \sigma\left(p_{1}, p_{2}, . ., p_{k-2}, p_{k-1}\right)\left\|M_{1}(t) x\right\|^{2},
$$

where

$$
\sigma\left(p_{1}, p_{2}, . ., p_{k-2}, p_{k-1}\right)=\frac{\left(p_{1}-\alpha\right) \sigma\left(p_{2}, . ., p_{k-2}, p_{k-1}\right)-\alpha^{2}}{2\left(p_{1}-\alpha+\sigma\left(p_{2}, . ., p_{k-2}, p_{k-1}\right)\right)} .
$$

Let us denote $M_{1}(t) x=y$. Since the matrix $M_{1}(t)$ is non-degenerate then we get $\left\langle L\left[S_{p}\right] y, y\right\rangle \geq \sigma\left(p_{1}, p_{2}, . ., p_{k-2}, p_{k-1}\right)\|y\|^{2}$ from the inequality (2.14) for any $y \in R^{n}$ with positive coefficient $\sigma\left(p_{1}, p_{2}, . ., p_{k-2}, p_{k-1}\right)$. That means the derivative of the quadratic form $V_{p}$ with respect to the system $(2.1)$ at certain choices of the vector of parameters $\left(p_{1}, p_{2}, . ., p_{k-2}, p_{k-1}\right)=p$ will be positive definite. The Theorem 2.5 is proved.

Let us consider an example of the application of the proved theorem.

Denote

$$
a(t ; \lambda)=\frac{\lambda e^{-t}-(1-\lambda) e^{t}}{\lambda e^{-t}+(1-\lambda) e^{t}}, 0 \leq \lambda \leq 1
$$

and consider the system

$$
\left\{\begin{array}{c}
\frac{d x_{1}}{d t}=\left[a\left(t ; \lambda_{1}\right)+a\left(t ; \lambda_{2}\right)-1\right] x_{1}+\left[a\left(t ; \lambda_{1}\right)+a\left(t ; \lambda_{2}\right)\right] x_{2}, \\
\frac{d x_{2}}{d t}=\left[-a\left(t ; \lambda_{2}\right)+1\right] x_{1}-a\left(t ; \lambda_{2}\right) x_{2}, \\
\frac{d x_{3}}{d t}=\left[a\left(t ; \lambda_{2}\right)+1\right] x_{1}-\left[a\left(t ; \lambda_{1}\right)+a\left(t ; \lambda_{2}\right)\right] x_{2}-a\left(t ; \lambda_{1}\right) x_{3},
\end{array}\right.
$$

where $\lambda_{1}, \lambda_{2}$-independent parameters from the closed segment $[0,1]$.

We choose the matrices $S_{i}, M_{i}$ in the following form

$$
\begin{aligned}
& S_{1}=\left(\begin{array}{ccc}
0 & 1 & 1 \\
1 & 0 & 1 \\
1 & 1 & 0
\end{array}\right) \quad, \quad S_{2}=\left(\begin{array}{ccc}
0 & 0 & 0 \\
0 & -a\left(t ; \lambda_{2}\right) & 0 \\
0 & 0 & 0
\end{array}\right), \quad S_{3}=\left(\begin{array}{ccc}
0 & 0 & 0 \\
0 & 0 & 0 \\
0 & 0 & -a\left(t ; \lambda_{1}\right)
\end{array}\right), \\
& M_{1}=\left(\begin{array}{lll}
1 & 0 & 0 \\
0 & 1 & 0 \\
0 & 0 & 1
\end{array}\right), \quad M_{2}=\left(\begin{array}{lll}
0 & 0 & 0 \\
0 & 1 & 0 \\
0 & 0 & 1
\end{array}\right), \quad M_{3}=\left(\begin{array}{lll}
0 & 0 & 0 \\
0 & 0 & 0 \\
0 & 0 & 1
\end{array}\right) \text {. }
\end{aligned}
$$

Calculating the left sides of the inequalities (2.11) and (2.12) $(k=3)$, we get

$$
\begin{gathered}
L\left[S_{1}\right]=\left(\begin{array}{ccc}
2 & 0 & 0 \\
0 & 0 & 0 \\
0 & 0 & 0
\end{array}\right), L\left[S_{2}\right]=\left(\begin{array}{ccc}
0 & \left(a^{2}\left(t ; \lambda_{2}\right)-a\left(t ; \lambda_{2}\right)\right) & 0 \\
\left(a^{2}\left(t ; \lambda_{2}\right)-a\left(t ; \lambda_{2}\right)\right) & \left(2 a^{2}\left(t ; \lambda_{2}\right)-\frac{d a\left(t ; \lambda_{2}\right)}{d t}\right) & 0 \\
0 & 0
\end{array}\right), \\
L\left[S_{3}\right]=\left(\begin{array}{ccc}
0 & -a\left(t ; \lambda_{1}\right)\left[a\left(t ; \lambda_{2}\right)+1\right] \\
0 & a\left(t ; \lambda_{1}\right)\left[a\left(t ; \lambda_{1}\right)+a\left(t ; \lambda_{2}\right)\right] \\
-a\left(t ; \lambda_{1}\right)\left[a\left(t ; \lambda_{2}\right)+1\right] & a\left(t ; \lambda_{1}\right)\left[a\left(t ; \lambda_{1}\right)+a\left(t ; \lambda_{2}\right)\right] & \left(2 a^{2}\left(t ; \lambda_{1}\right)-\frac{d a\left(t ; \lambda_{1}\right)}{d t}\right)
\end{array}\right) .
\end{gathered}
$$

Hence it is already clear that the inequalities (2.11) and (2.12) are fulfilled. Thus, the derivative of the quadratic form $p_{1}\left(x_{1} x_{2}+x_{1} x_{3}+x_{2} x_{3}\right)-p_{2} x_{2}^{2} a\left(t ; \lambda_{2}\right)-x_{3}^{2} a\left(t ; \lambda_{1}\right)$ with respect to the system $(2.15)$ with the appropriate choice of parameters $p_{1}, p_{2}$ will be positively defined. This implies that the system (2.15) is regular on $R$. 


\section{References}

[1] Yu. A. Mitropolsky, A. M. Samoilenko, V. L. Kulik, Dichotomies and Stability in Non-Autonomous Linear Systems, Taylor\&Francis, London, 2003.

[2] V. Kulyk, D. Poczko, Some conditions of regularity of linear extensions of dynamical systems with respect to selected variables, Nonlinear Anal. Model. Control, 19(4) (2014), 602-610.

[3] V. L. Kulyk, N. V. Stepanenko, Alternating Lyapunov's functions in the theory of linear extensions of dynamical systems on a torus, Ukrainian Math. J., 59(4) (2007), 546-562.

[4] V. L. Kulyk, G. N. Kulyk, N. V. Stepanenko, Addition of weakly regular linear extensions of dynamical systems to regular, Almaty, 11(1) (2011), 74-86. 